\title{
A Dynamic QoS Adaptation Mechanism for Networked Virtual Reality
}

Seiwoong Oh* $^{*}$ Hiroyuki Sugano*, Kazutoshi Fujikawa ${ }^{\dagger}$

Toshio Matsuura ${ }^{\circ}$, Shinji Shimojo*, Masatoshi Arikawa $\ddagger$ and Hideo Miyahara*

* Department of Information and Mathematical Sciences, Graduate School of Engineering Science Osaka University, Toyonaka, Osaka 560, Japan

Tel: +81-6-850-6588 Fax: +81-6-850-6589

$\dagger$ Graduate School of Information Science Nara Institute of Science and Technology

- Osaka City University

$\ddagger$ Hiroshima City University

\{osw,h-sugano,shimojo,miyahara\}@ics.es.osaka-u.ac.jp,

fujikawa@is.aist-nara.ac.jp, matsuura@media.osaka-cu.ac.jp, arikawa@its.hiroshima-cu.ac.jp

\section{Abstract}

In this paper, we propose a dynamic QoS adaptation mechanism for the networked virtual reality (VR) system to improve responsiveness to the users and introduce a live video into an networked VR system. To keep presentation quality as much as possible from point of view of user's perception when network and local system resources are going to starve, we introduce the notion of an importance of presence $(I o P)$ of objects. In case of the resource starvation, our VR system reduces QoSs of both Computer Graphic (CG) objects and Video objects based on IoP. That is, the quality of the object whose IoP is the lowest in the virtual space is reduced.

\section{Keywords}

Networked virtual reality, QoS control, QoS adaptation

\section{INTRODUCTION}

Soon after the invention of virtual reality where people can navigate through computer generated graphic virtual spaces, they can share virtual spaces through exchanging their descriptions of virtual spaces by using Virtual Reality Modeling Language (VRML)(Pesce 1995)(Hagsand 1996).

However, although one can move from a virtual space to another one by just one click, he must wait for a while until an entire file describing the

Building QoS into Distributed Systems A. Campbell \& K. Nahrstedt (Eds.)

(C) 1997 IFIP. Published by Chapman \& Hall 
virtual space is retrieved and a new virtual space is constructed on his local client. As a result, a smooth navigation among virtual spaces suffers from file transfers whenever one move to another virtual space. That is, the quality of the navigation is greatly decreased by the time spent for transferring the file and constructing the incoming virtual space. Although transferring an entire file at the start avoids uncertainty of best effort network performance, that is, network Quality of Service (QoS), it makes a large virtual space model intractable and loses responsiveness to the user during navigating a virtual space.

In addition to this problem, an introduction of a live video into a virtual space may cause another problem. By introducing a live video into a virtual space, one can bring more realistic elements into the virtual space such as a face image of an attendee in the virtual meeting room or an actual outside scenery shown in a virtual window (Arikawa et al. 1996). In this case, the quality of the video is greatly affected by the currently available network QoS such as bandwidth, delay and jitter, and amounts of processing power for rendering video frames on the screen. During the execution of the system, these resources may starve, and in such a case the system can not maintain the quality of the virtual space. Therefore, it is necessary to introduce an mechanism which adapts a quality of a VR system to the currently available CPU and network resources. In this paper, we call such a mechanism the dynamic QoS adaptation mechanism.

Other related works focusing on VR systems (Pesce 1995)(Arikawa et al. 1996)(Nakanishi et al. 1996) proposed QoS control models based on the level of detail (LoD), which considers only the distance between objects and user. Moreover, these models never consider streaming objects for smooth navigation.

\section{THE DYNAMIC QOS ADAPTATION MECHANISM}

Our purpose is the seamless integration of a live video with VR, as well as the incremental acquisition of a large VR space. For incremental acquisition of a large VR space, we propose a mechanism called the object streaming which can handle a large space interactively and deal with live media such as streaming video. And also, for the seamless integration of VR with a live video, we apply the notion of $I o P$ (Importance of Presence) to the video objects as well as the CG objects. When the network and local resources are going to starve, the proposed system copes with the starvation by reducing the quality of the object whose IoP is the lowest.

In the IoP of the CG object is derived as follows:

$I o P=100 \times\left(1-\frac{l}{L}\right) \times \cos \theta$ 


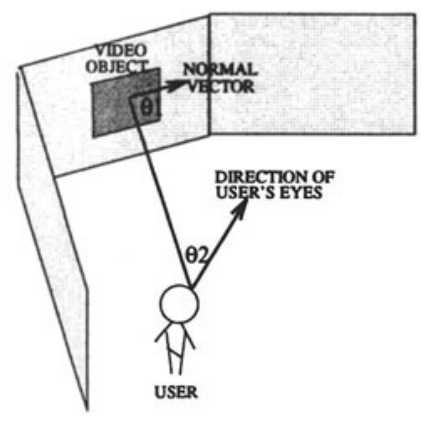

Figure 1 IoP of a video object

where $L, l$, and $\theta$ are the maximum visible distance in the virtual space, the distance between the user and the CG object, and the angle between the direction of the user's eyes and the direction of the CG object, respectively.

The $I o P$ of video objects is calculated as follows:

$I o P=100 \times S \times \cos \theta_{1} \times \cos \theta_{2}$

where $S, \theta_{1}$ and $\theta_{2}$ represent the ratio of the area occupied by the video object to the whole display area, the angle between the normal vector of the surface of the video object and the direction of the gravity of the video object with respect to the user's position, and the angle between the direction of the user's eyes and the direction of the gravity of the video object, respectively (Figure. 1).

The proposed mechanism controls the LoD of each object based on its $I o P$ when the system is going to starve. For the video objects, the proposed mechanism assigns units of CPU time and network bandwidth in proportion to their IoPs and then adjusts the temporal and spatial resolution to fit into the assigned units.

Since the evaluation of IoPs of objects and the decision of what objects are sent in object streaming may cause some delay at the client, the navigation may not work smoothly. For the smooth navigation through a VR space, Funkhouser(Funkhouser 1996) proposed a prediction mechanism for reducing the delay of disk-to-memory copy. We apply a similar mechanism to the client/server environment. The prediction is expected to work effectively in VR system because a movement of a user in the VR space has continuity. For the prediction, the client periodically sends the user's current position, the direction of his viewpoint, and the type of his motion(straight, rotation) to the server.

Figure. 2 shows the overview of system with the proposed mechanism. 


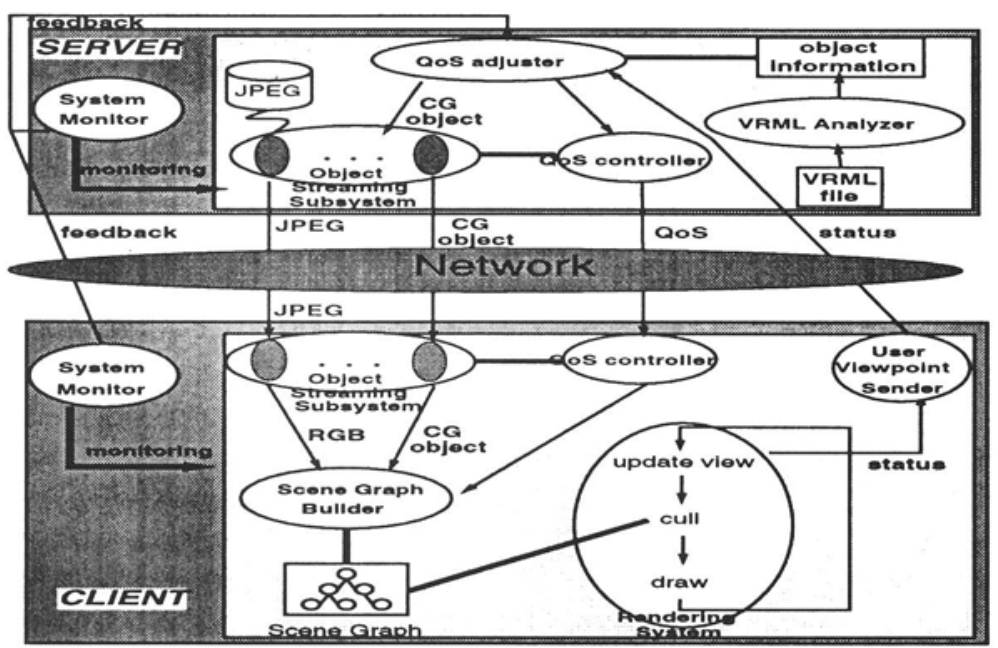

Figure 2 The overview of the prototype virtual reality system

\section{CONCLUSION}

In this paper, a dynamic QoS adaptation mechanism is presented for the seamless integration of VR and a live video and the incremental acquisition of a large VR space. Experimental results on the SGI Indigo2 show that the VR system with the proposed mechanism is efficient in respect of smooth navigation and smaller user waiting time.

\section{REFERENCES}

M. Pesce (1995) VRML - Browsing and Building Cyberspace, New Riders.

O. Hagsand (1996) Interactive Multiuser VEs in the DIVE System, IEEE MultiMedia, pp. 30-38.

M. Arikawa, A. Amano, K. Maeda, R. Aibara, S. Shimojo, Y. Nakamura, K. Hiraki, K. Nishimura, and M. Terauchi (1996) Dynamic LoD for QoS Management in the Next Generation VRML, IEEE,Proc. of the International Conference on Multimedia Computing and Systems, pp. 24-27.

H. Nakanishi, C. Yoshida, T. Nishimura, and T. Ishida (1996) Freewalk:Supporting Casual Meetings in a Network, International Conference on Computer Supported Cooperative Work, pp. 308-314.

Thomas A. Funkhouser (1996) Database Management for Interactive Display of Large Architectural Models, Graphics Interface'96, Toronto, Ontario , pp. 1-8. 